\title{
A New Resonance Prediction Method of Fabry-Perot Cavity (FPC) Antennas Enclosed with Metallic Side Walls
}

\author{
Dongho $\mathrm{Kim}^{1} \cdot$ Junho $\mathrm{Yeo}^{2}$
}

\begin{abstract}
We have proposed a new method to accurately predict the resonance of Fabry-Perot Cavity (FPC) antennas enclosed with conducting side walls. When lateral directions of an FPC antenna are not blocked with metallic walls, the conventional technique is accurate enough to predict the resonance of the FPC antenna. However, when the FPC antenna has side walls, especially for case with only a short distance between the walls, the conventional prediction method yields an inaccurate result, inevitably requiring a tedious, time-consuming tuning process to determine the correct resonant height to provide the maximum antenna gain in a target frequency band using three-dimensional full-wave computer simulations. To solve that problem, we have proposed a new resonance prediction method to provide a more accurate resonant height calculation of FPC antennas by using the well-known resonance behavior of a rectangular resonant cavity. For a more physically insightful explanation of the new prediction formula, we have reinvestigated our proposal using a wave propagation characteristic in a hollow rectangular waveguide, which clearly confirms our approach. By applying the proposed technique to an FPC antenna covered with a partially reflecting superstrate consisting of continuously tapered meander loops, we have proved that our method is very accurate and readily applicable to various types of FPC antennas with lateral walls. Experimental result confirms the validness of our approach.
\end{abstract}

Key words: Fabry-Perot Cavity, Resonance, Antenna, Prediction Formula.

\section{I . Introduction}

In the history of antenna research and development, enhancement of antenna gain has continuously been an area of focus for a variety of research groups. Using cavities surrounding antennas is one solution to efficiently increase antenna gain. Fabry-Perot cavity (FPC), electromagnetic band gap (EBG) or partially reflecting surface (PRS) superstrate are all categorized into cavity-type antennas [1] [5].

A detailed method of computing a cavity resonance condition to obtain maximum antenna gain was analyzed by Trentini [6]. Trentini described directivity with a reflection coefficient of a partially reflecting superstrate, a wave traveling distance in a cavity composed of the superstrate and a ground plane, where a resonant height, i.e. the cavity height can be determined from the phase of the reflection coefficients of the superstrate and the ground plane.

The conventional resonance prediction technique described by Trentini [6] is very easy and accurate for predicting an antenna's resonant height. Therefore, it is not hard to find various successful examples of applying the conventional technique [7] [10]. However, when lateral directions of the antenna are enclosed with metallic side walls, a prediction result obtained from the conventional resonance prediction method has a large error. In general, the shorter the distance between the side walls yields the larger the error in prediction of a resonant height.

In this paper, we have proposed a simple but precise way to accurately predict the resonant heights of antennas consisting of partially reflecting superstrates and substrates such as FPCs, EBGs, and PRSs.

To examine the validity of our approach, we fabricated a somewhat complicated PRS superstrate composed of square meander copper loops of similar figures, which consists of an FPC antenna. The experimental data proved that the predicted resonant height is much more accurate than that obtained using the conventional technique, which confirms the usefulness of our approach.

\section{II . Conventional Prediction Technique}

The operational principle to increase antenna gain using

\footnotetext{
Manuscript received May 27, 2011 ; revised August 26, 2011. (ID No. 20110527-01J)

${ }^{1}$ Department of Electronic Engineering, Sejong University, Seoul, Korea.

${ }^{2}$ School of Computer \& Communication Engineering, Daegu University, Gyeongbuk, Korea.

Corresponding Author : Junho Yeo (e-mail : jyeo@daegu.ac.kr)
} 


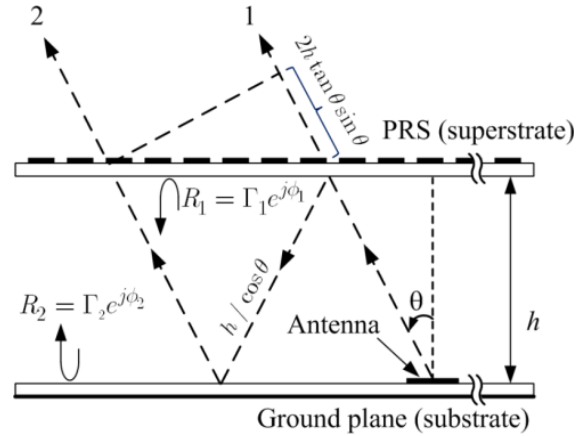

Fig. 1. Principle of increasing antenna gain using cavity resonance, which is composed of the substrate and the superstrate.

a cavity consisting of a substrate and a superstrate is depicted in Fig. 1. In general, the superstrate is a PRS and the substrate is a ground plane, therefore, the reflection phase $\left(\phi_{2}\right)$ of the substrate is normally close to $180^{\circ}$. The reflection phase $\left(\phi_{1}\right)$ of the superstrate is largely dependent on the unit cell geometry of the superstrate. In other words, antenna designers can obtain an arbitrary value of $\phi_{1}$ by selecting appropriate geometry of unit cells and changing sizes of the unit cells.

In Fig. 1, a trajectory of only one ray is shown, which has a launching angle of $\theta$ from the antenna placed right on the ground plane. We can find two individual radiating paths of 1 and 2 , and the phase difference $\left(\Theta_{1}\right)$ between them is computed as

$$
\begin{aligned}
\Theta_{1} & =2 k_{0} h \tan \theta \sin \theta-\frac{2 k_{0} h}{\cos \theta}+\phi_{1}+\phi_{2} \\
& =2 k_{0} h\left(\frac{\tan ^{2} \theta-1}{\cos \theta}\right)+\phi_{1}+\phi_{2},
\end{aligned}
$$

where $k_{0}$ is a wave propagation constant in free space. Let us assume that the substrate is a normal ground plane, which is a general case in numerous antenna applications. After some mathematical manipulations, directivity of the antenna can be written as [6]:

$$
D=\frac{1-\Gamma_{1}^{2}}{1+\Gamma_{1}^{2}-2 \Gamma_{1} \cos \left(\phi_{1}-\pi-2 k_{0} h \cos \theta\right)} .
$$

The maximum directivity in the antenna's normal direction $(\theta=0)$ presents a condition of cavity resonance, which determines the resonant height $(h)$ as following:

$$
h=\frac{\lambda}{4}\left(\frac{\phi_{1}}{\pi}-1\right)+N \frac{\lambda}{2},
$$

where $N$ is an integer and $\lambda$ is a free space wavelength. That is to say, if we satisfy the resonant height derived in eq. (3), then we can obtain maximum antenna gain in a normal direction of the antenna.

Actually, eq. (3) is very simple and accurate for predicting antenna heights, which is fairly helpful for saving time required for a somewhat complicated threedimensional fine tuning process.

\section{Advanced Prediction Technique}

In general, the cavity shown in Fig. 1 has only two walls of the substrate and the superstrate. In that case, a portion of energy inevitably leaks out of the cavity through the openings in lateral directions, which causes an unnecessary gain drop. To prevent the leakage and to raise the antenna gain, we can block all openings in the lateral directions with metallic walls. Then, now the cavity is completely enclosed with six walls, i.e. four metallic side walls, one bottom ground plane, and one top PRS.

At this point, we need to check the validity of our prediction method to determine whether eq. (3) is accurate enough to predict the resonant height while providing the maximum gain. If the ground plane and the PRS are larger than several wavelengths, then eq. (3) is valid. However, when the distances between side walls facing each other are shorter than one or two times the wavelength, then eq. (3) does not provide an accurate prediction. The prediction error is inversely proportional to the distance between the side walls.

In this case, we need a different approach for a more accurate prediction, which can take the effect of newly installed metallic side walls into account. In previous research [11], we proposed a new prediction formula modified from the resonance property of a rectangular resonant cavity [12], in which coupling effects introduced by side walls are included as follows:

$$
\begin{aligned}
& f_{m n q}=\frac{c}{2 \pi} \sqrt{\beta_{x}^{2}+\beta_{y}^{2}+\beta_{q}^{2}} \\
& \text { where } \beta_{x}=\frac{m \pi}{l_{x}}, \beta_{y}=\frac{n \pi}{l_{y}}, \text { and } \beta_{q}=\frac{2 \pi f_{q}}{c} .
\end{aligned}
$$

In eq. (4), $\beta_{x}, \beta_{y}$, and $\beta_{q}$ are eigen wave numbers, which determine eigen-modes in each direction. Here, $f_{q}$ is a resonant frequency of the cavity, and is given by

$$
f_{q}=\frac{c}{2 h}\left[\frac{\phi_{h}+\phi_{l}}{2 \pi}+q\right], q=0,1,2, \cdots
$$

where $\phi_{h}$ and $\phi_{l}$ are reflection phases of the superstrate and the substrate, respectively. Combining eqs. (4) and (5) gives a resonant height $(h)$ of the cavity antenna (i.e. the FPC antenna), which is written as 


$$
h=\frac{\left(\phi_{h}+\phi_{l}+2 \pi q\right)}{2 \sqrt{k_{0}^{2}-\left(\beta_{x}^{2}+\beta_{y}^{2}\right)}}
$$

Comparing eq. (3) with eq. (6) tells us that the correction factor of $\beta_{x}^{2}+\beta_{y}^{2}$ is newly inserted in eq. (6), which can handle the possible eigen-modes generated in the cavity. Therefore, we can determine the resonant height more accurately with the help of eq. (6).

Eq. (6) was derived using a basic principle of resonance in a rectangular cavity. However, considering the wave propagation behavior in a hollow rectangular waveguide presents us the same result derived in eq. (6), which is fairly important from a physical point of view.

In eq. (1), the first term is the phase difference caused by the different paths of 1 and 2 outside the cavity. However, the second term came from the wave traveling path in the cavity. Therefore, the wave number $k_{0}$ should be corrected to another quantity that satisfies a dispersion relation in the cavity. At this point, we can consider the cavity a hollow waveguide supporting a slow wave traveling toward the $\mathrm{z}$-direction, which is enclosed with metallic walls both in the $\mathrm{x}$ - and $\mathrm{y}$-directions. Therefore, $k_{0}$ the second term of eq. (1) should be replaced with $k_{r}$, satisfying a wave equation in the waveguide, which is written as

$$
k_{r}=\sqrt{k_{0}^{2}-\beta_{x}^{2}-\beta_{y}^{2}},
$$

where $\beta_{x}$ and $\beta_{y}$ are given in eq. (4). Consequently, eq. (1) should be corrected as

$$
\begin{aligned}
\Theta_{1} & =2 k_{0} h \tan \theta \sin \theta-\frac{2 k_{r} h}{\cos \theta}+\phi_{1}+\phi_{2} \\
& =2 h\left(\frac{k_{0} \tan ^{2} \theta-k_{r}}{\cos \theta}\right)+\phi_{1}+\phi_{2},
\end{aligned}
$$

Inserting eq. (7) into eq. (8) gives a generalized expression of directivity written as

$$
D=\frac{1-\Gamma_{1}^{2}}{1+\Gamma_{1}^{2} \Gamma_{2}^{2}-2 \Gamma_{1} \Gamma_{2} \cos \left(\phi_{1}+\phi_{2}-2 k_{r} h \cos \theta\right)}
$$

From the maximum directivity at $\theta=0, \phi_{h}+\phi_{l}-$ $2 k_{r} h \cos \theta$ should be an integer multiple of $2 \pi$ to minimize the denominator of eq. (9), which results in exactly the same result relationship written in eq. (6).

It is very important to note that the derivation of the modified resonant height $h$ in eq. (6) from investigating boundary conditions of a hollow rectangular waveguide is not astonishing but quite natural, because the wave properties such as a wavelength and propagation velocity should obey wave characteristics in a hollow wave- guide.

To examine the exactness of eq. (6) or eq. (9), we fabricated an FPC antenna. Its superstrate and unit cell geometry are shown in Fig. 2. The unit cell is composed of square copper meander-loops etched on one side of $1.57 \mathrm{~mm}$ thick dielectric laminate with a relative permittivity of 2.2. For a more complicated situation for predicting antenna resonance, the superstrate is composed of tapered unit cells, which have different sizes in different columns as shown in Fig. 2.

First, we computed each reflection phase of the superstrate and the substrate. One of them is shown in Fig. 3(a). The black solid line is for the biggest loops in the first column, and the red broken line is for the ground plane. Because we have used a ground plane covered with a $1.57 \mathrm{~mm}$ thick dielectric layer with relative permittivity of 3.0 , the reflection phase is a bit smaller than

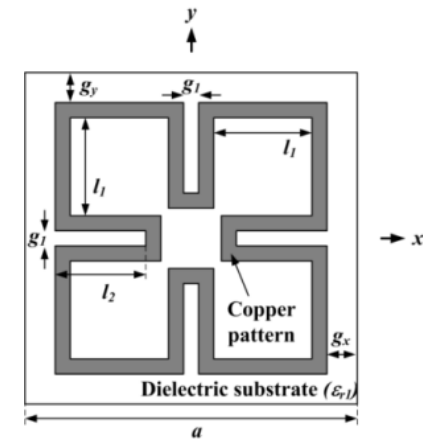

(a) Unit cell

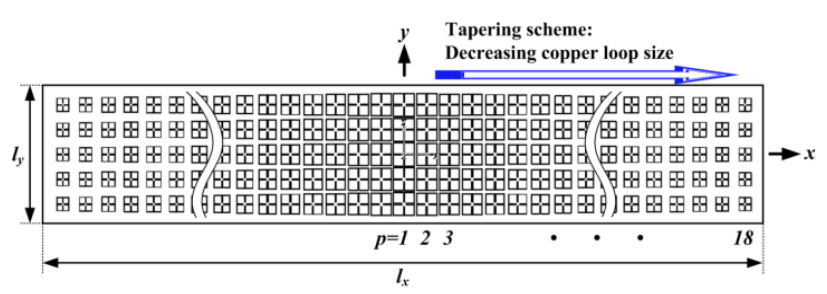

(b) Superstrate

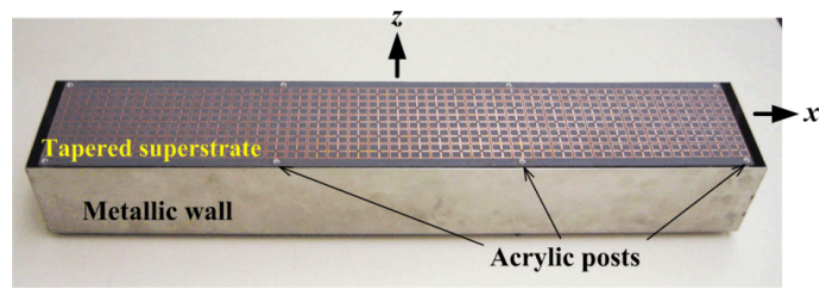

(c) Fabricated antenna

Fig. 2. (a) Proposed unit cell structure with the biggest meander copper loop with $a=17 \mathrm{~mm}, l_{1}=6.95 \mathrm{~mm}$, $l_{2}=6.1 \mathrm{~mm}, g_{1}=0.21 \mathrm{~mm}$, and $g_{x}=g_{y}=0.4 \mathrm{~mm}$, (b) Unit cell tapering scheme on the superstrate for wider radiation bandwidth with $l_{x}=615 \mathrm{~mm}$ and $l_{y}=105 \mathrm{~mm}$, and (c) a photo of a fabricated antenna. 


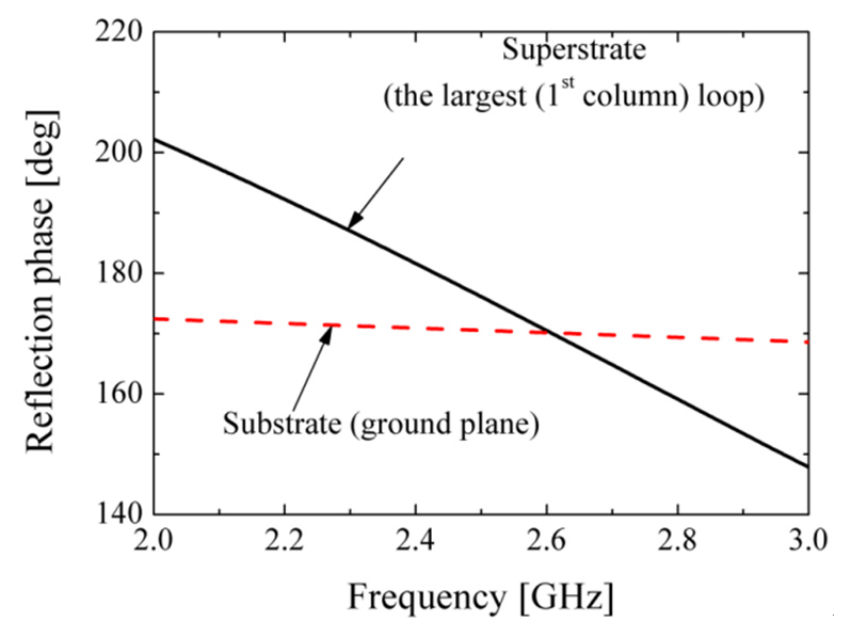

(a) Reflection phase

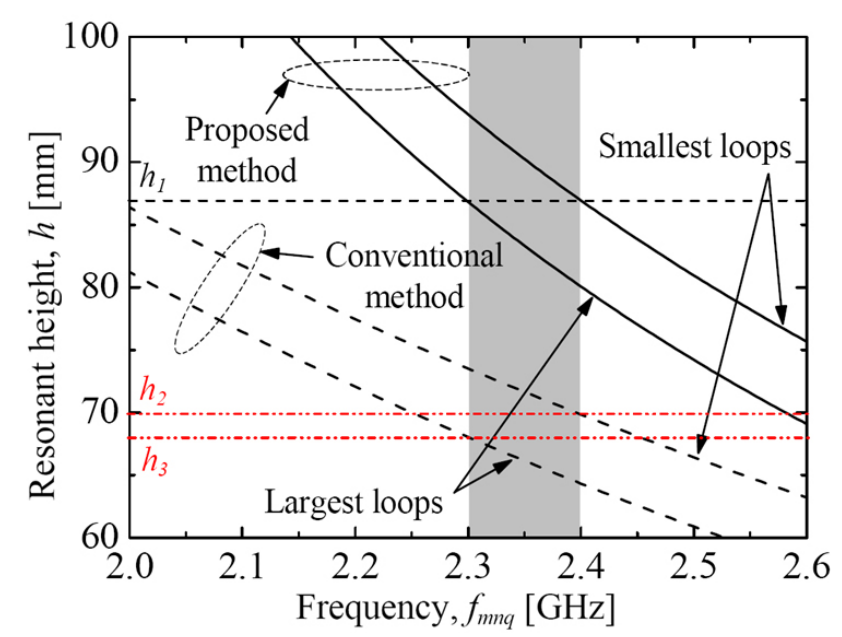

(b) Predicted resonant heights

Fig. 3. (a) Reflection phase of the superstrate and the substrate, and (b) Comparison of a predicted resonant height using the conventional and the proposed method.

$180^{\circ}$. Substituting these phase values into eq. (3) and eq. (6), we can determine a resonant height, which will provide the highest gain in a normal direction.

Different prediction results obtained by using the conventional and the proposed method are compared in Fig. 3. As for the largest loops in the first column $(p=1)$ of the superstrate shown in Fig. 2, the conventional method presents $h_{3}=68 \mathrm{~mm}$. For the smallest loops that have a size of $92 \%$ of the largest loops, it predicts $h_{2}=70 \mathrm{~mm}$. The proposed method results in $h_{1}=86 \mathrm{~mm}$ for the largest and the smallest loops, which has a large discrepancy with the results determined from the conventional way. Through the simulation and experiment, we determined that the resonant height of $h=82.8 \mathrm{~mm}$ gives the maximum gain and the radiation bandwidth in the target WiBro frequency band.

There is an alternative way to predict the resonant

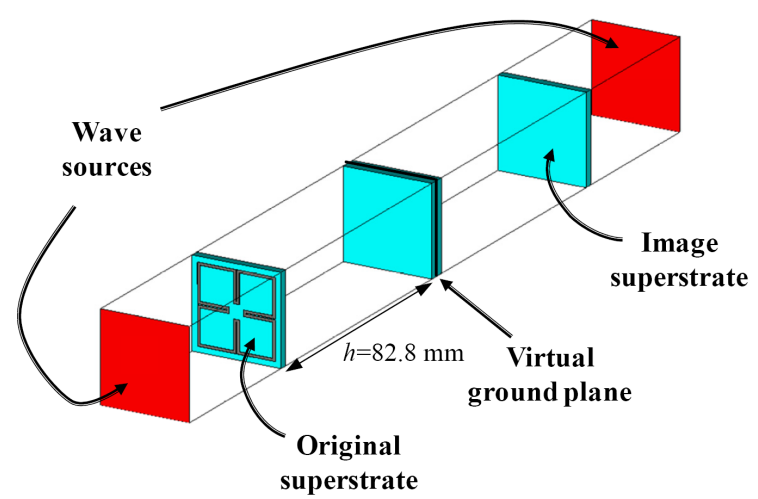

(a) Image theory

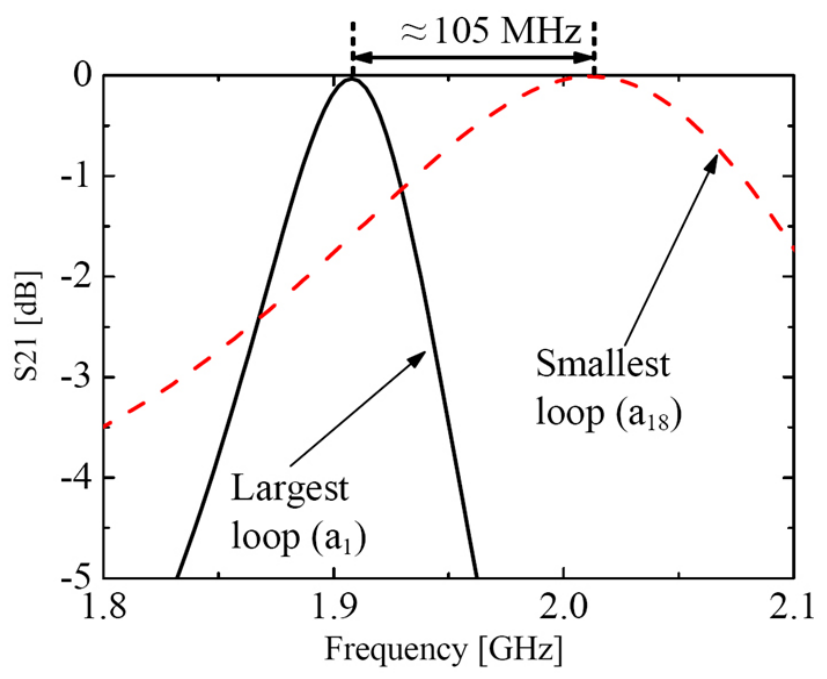

(b) Prediction result of the image theory

Fig. 4. (a) Applying image theory, and (b) Resonant height predicted using the image theory.

height using image theory, which is depicted in Fig. 4(a). This method forms a fictitious image structure of the superstrate beyond the ground plane, removes the ground plane, and finds the distance that gives the highest transmission at the required frequency point. The prediction result using the image theory is shown in Fig. 4(b), which provides resonant frequencies ranging from about $1.9 \mathrm{GHz}$ to $2.0 \mathrm{GHz}$. Therefore, we can conclude that the alternative image theory is much more inaccurate compared to the proposed formula. To see the prediction error quantitatively, we compared the maximum directivity obtained from both the conventional formula and the proposed formula, which is shown in Fig. 5. In Fig. 5, we can see not only the frequency difference but also the magnitude of directivity, which is very helpful to get the feel for antenna operation. Because all the formulas are based on an infinitely large antenna, the directivity values shown in Fig. 5 are much greater than real values, which is not possible to obtain with a proposed size of the antenna. 


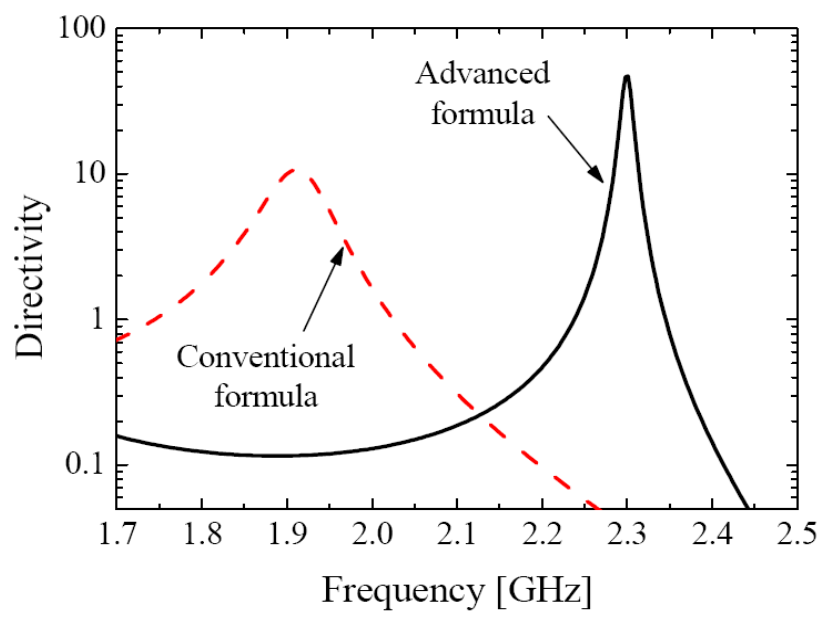

Fig. 5. Comparison of directivity computed from the conventional and the proposed formula for $h=82.8 \mathrm{~mm}$.

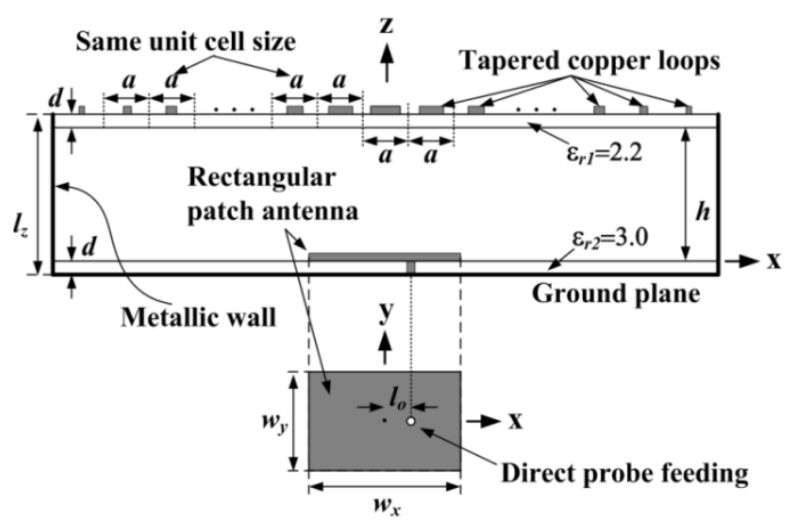

Fig. 6. Fabricated FPC antenna with $a=17 \mathrm{~mm}, d=1.57 \mathrm{~mm}$, $w_{x}=35 \mathrm{~mm}, w_{y}=21 \mathrm{~mm}, l_{o}=7 \mathrm{~mm}, l_{z}=86 \mathrm{~mm}$, and $h=$ $82.8 \mathrm{~mm}$.

To investigate the validity of our approach, we have fabricated an FPC antenna, which is shown in Fig. 6. For an initial wave launching from the bottom of the FPC, we have used a direct probe-fed rectangular micro strip patch antenna. To obtain a wide radiation bandwidth and high gain, the superstrate is composed of tapered unit cells decreasing from the center column $(p=1)$ toward the edge column $(p=18)$ and the resonant height $h$ is fixed to $82.8 \mathrm{~mm}$ after a fine-tuning step through some numerical simulations.

The input reflection coefficient $\left(S_{11}\right)$ is compared with the measured data, as shown in Fig. 7. As for $S_{11}$, the first measured impedance matched frequency relatively well with the prediction. However, the second frequency point shows discrepancy of about $50 \mathrm{MHz}$, which might be caused from an unexpected gap between the side walls and the superstrate generated from imperfect fabrication.

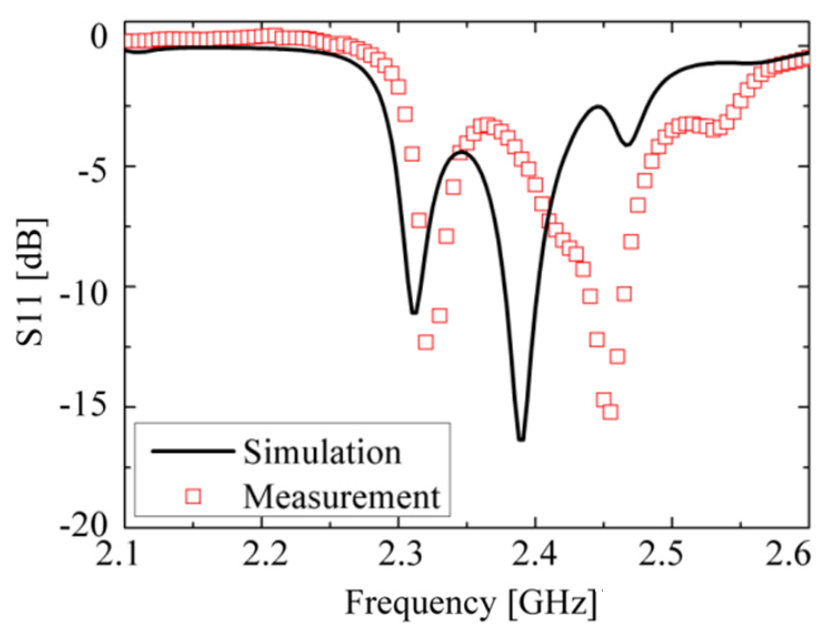

(a) Input reflectoin coefficient $\left(S_{11}\right)$

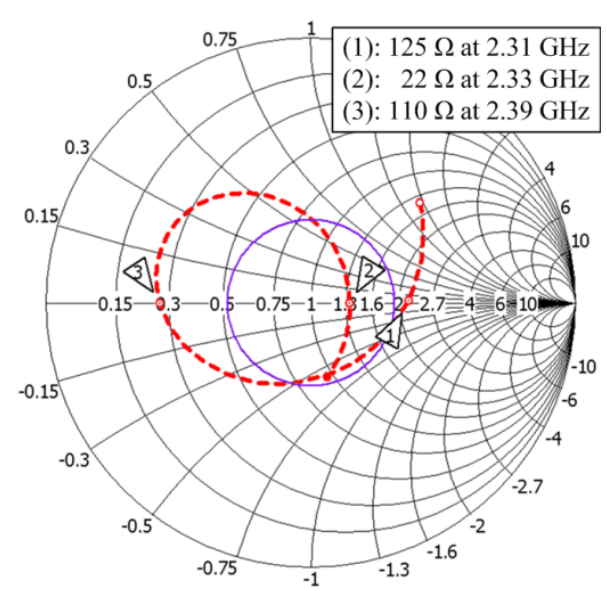

(b) Impedance variation on Smith chart

Fig. 7. Impedance-matching properties.

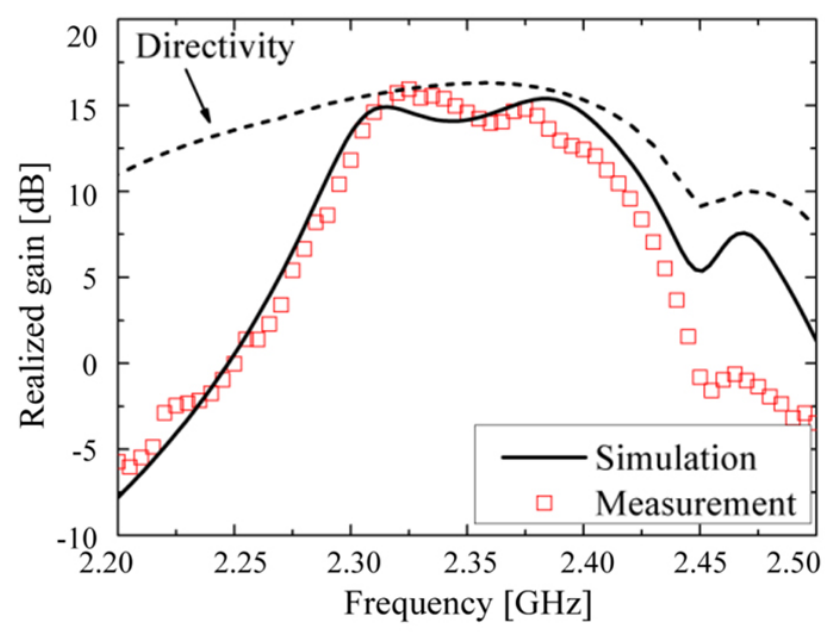

Fig. 8. Comparison of measured and predicted realized gain.

Impedance matching properties (shown in Fig. 7(a)) can be analyzed in more detail using the Smith chart presented in Fig. 7(b). Three points on the Smith chart 
correspond to the frequencies of zero reactance. The values at three frequency points shown in Fig. 7(b) are matched well with $S_{11}$ property shown in Fig. 7(a).

Predicted realized gain was also compared with experimental data (see in Fig. 8). Regarding realized gain, two data show relatively good agreement. As shown in Fig. 6, by using continuous tapering in cell sizes, we can obtain relatively flat gain values in the target frequency band. This behavior can be expected from resonance prediction result given in Fig. 3(b), which satisfies an FPC resonance condition in the whole target frequency band.

\section{Conclusion}

We have proposed a new method to predict the resonant height of an FPC antenna enclosed with metallic walls. By introducing resonance behavior of a rectangular resonant cavity to the new prediction formula, we can increase the accuracy of resonance prediction, which provides much a more precise result than that from the conventional approach. In addition, by investigating the new formula by analyzing a dispersion relation in a hollow rectangular waveguide, we can provide a solid physical background of the proposed formula. Therefore, with the help of the new approach, we can design FPC antennas faster and easier, which is obviously advantageous from a practical point of view.

\section{References}

[1] D. Kim, J. Choi, "Analysis of antenna gain enhancement with a new planar metamaterial superstrate: en effective medium and a Fabry-Perot resonance approach," J. Infrared Milli. Terahz Waves, vol. 31, no. 11, pp. 1289-1303, 2010.

[2] N. Guerin, S. Enoch, G. Tayeb. P. Sabouroux, P. Vincent, and H. Legay, "A metallic fabry-perot directive antenna," IEEE Trans. Antennas Propag., vol. 54, no. 1, pp. 220-224, 2006.
[3] E. Rodes, M. Diblanc, E. Arnaud, T. Monediere, and B. Jecko, "Dual-band EBG resonator antenna using a single-layer FSS," IEEE Antennas Wireless Propag. Lett., vol. 6, pp. 368-371, 2007.

[4] A. Pirhadi, M. Hakkak, F. Keshmiri, and R. K. Bae, "Design of compact dual band high directive electromagnetic bandgap (EBG) resonator antenna using artificial magnetic conductor," IEEE Trans. Antennas Propag., vol. 55, no. 6, pp. 1682-1690, 2007.

[5] A. P. Feresidis, J. C. Vardaxoglou, "High gain planar antenna using optimised partially reflecting surfaces," IEE Proc Microwave Antennas Propag., vol. 148, no. 6, pp. 345-350, 2001.

[6] G. V. Trentini, "Partially reflecting sheet arrays," IRE Trans Antennas Propag., vol. 4, no. 4, pp. 666-671, 1956.

[7] A. Ourir, S. N. Burokur, and A. Lustrac, "Bidimensional phase-varying metamaterial for steering beam antenna," Proc. of SPIE, vol. 6581, p. 65810R, 2007.

[8] Y. J. Lee, J. Yeo, R. Mittra, and W. S. Park, "Application of electromagnetic bandgap (EBG) superstrates with controllable defects for a class of patch antennas as spatial angular filters," IEEE Trans. Antennas Propagat., vol. 55, no. 1, pp. 224-235, 2005.

[9] H. Boutayeb, T. A. Denidni, "Internally excited Fabryperot type cavity power normalization and directivity evaluation," IEEE Antennas Wireless Propagat. Letts., vol. 5, pp. 159-162, 2006.

[10] G. K. Palikaras, A. P. Feresidis, and J. C. Vardaxoglou, "Cylindrical electromagnetic bandgap structures for directive base station antennas," IEEE Antennas Wireless Propagat. Letts., vol. 3, pp. 87-89, 2004.

[11] D. Kim, J. Ju, and J. Choi, "A broadband Fabryperot cavity antenna designed using an improved resonance prediction method," Microw. Opt. Tech. Lettes, vol. 53, no. 5, pp. 1065-1069, 2011.

[12] C. A. Balanis, Advanced Engineering Electromagnetics, John Wiley \& Sons, 1989. 


\section{Dongho Kim}

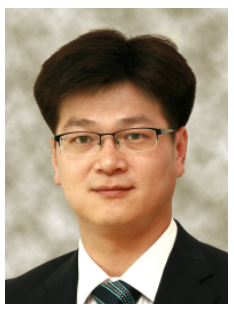

received the B.S. and M.S. degrees in electronics engineering from Kyungpook National University, Daegu, Korea, in 1998 and 2000, respectively, and the Ph.D. degree in electrical and electronics engineering from Korea Advanced Institute of Science and Technology (KAIST), Daejeon, Korea, in 2006. From 2000 to 2011, he was a Senior Researcher in Electronics and Telecommunications Research Institute (ETRI), Daejeon, Korea, where he was involved with the development of artificially engineered materials, metamaterials, and various antennas including RFID and mobile communication antennas. In 2011, he joined the Department of Electronic Engineering, Sejong University, Seoul, Korea, where he is now an Assistant Professor. His research interests include electromagnetic wave theory and application, design of highly-efficient and miniaturized antennas using artificially engineered materials, design of electromagnetic band-gap (EBG) structures, frequency selective surfaces (FSS), and artificial magnetic conductors (AMC), platform-tolerant special RFID antenna design, and development of a variety of metamaterials with negative permittivity and permeability. Prof. Kim is a member of the Institute of Electrical and Electronics Engineers (IEEE), and a life-member of the Korean Institute of Electromagnetic Engineering and Science (KIEES).

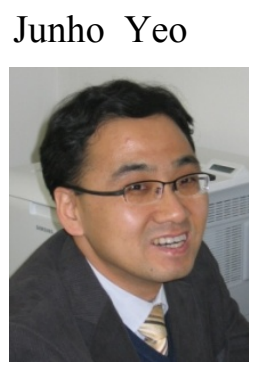

received Bachelor's and Master's degrees in electronics engineering from Kyungpook National University, Daegu, Korea, in 1992 and 1994, respectively, and the $\mathrm{Ph} . \mathrm{D}$. degree in electrical engineering from the Pennsylvania State University, University Park, in 2003. From 1994 to 1999, he was a researcher with the Republic of Korea Agency for Defense Development (ROKADD), Daejeon, Korea, where he was involved with the development of missile telemetry systems, especially the design and fabrication of low-profile transmitting and ground-station receiving antennas. From 1999 to 2003, he was a Graduate Research Assistant in the Electromagnetic Communication Laboratory (ECL), Pennsylvania State University. From September 2003 to June 2004, he was a Postdoctoral Research Scholar in the same laboratory. In August 2004, he joined Radio Frequency Identification (RFID) technology research team at Electronics and Telecommunications Research Institute (ETRI), Daejeon, Korea as a senior researcher. Since March 2007, he has been an Assistant Professor in the School of Computer and Communication Engineering at Daegu University, Gyeongsan, Korea. His research interests include computational electromagnetics, design of a class of antennas using electromagnetic bandgap (EBG) and artificial magnetic conductor (AMC) structures for RFID and mobile applications, portable wideband directive antenna design, and development of RFID sensor tags and long-range passive RFID tags. Prof. Yeo is a member of IEEE Wave Propagation Standards Committee and a reviewer for the IEEE Transaction on Antennas and Propagation, IET Microwaves, Antennas and Propagation, Progress in Electromagnetic Research (PIER), and ETRI Journal. He also holds a life membership of the Korea Institute of Electromagnetic Engineering and Science (KIEES) and serves as a reviewer. 